Research Article

\title{
Effects of Processed Polygonum multiflorum with KIOM Patent on Bone Remodeling-Related Protein Expression in Human Osteoblast-Like SaOS-2 Cells
}

\author{
Dae Uk Kim, ${ }^{1,2}$ Jae Yoon Chung, ${ }^{3}$ Seong Chul Jin, ${ }^{4}$ Mi Hye Kim, ${ }^{4}$ Richard Komakech, ${ }^{5,6,7}$ \\ Ki-Shuk Shim $\left(\mathbb{0},{ }^{8}\right.$ Yong-Goo Kim, ${ }^{5}$ Woong Mo Yang $\left({ }^{4},{ }^{4}\right.$ and Youngmin Kang $\mathbb{\circledR}^{5,6}$ \\ ${ }^{1}$ Oriental Pharmacy, College of Pharmacy, Woosuk University, Wanju-gun, Jeonbuk 55338, Republic of Korea \\ ${ }^{2}$ School of Korean Medicine, Pusan National University, Beomeo-ri, Mulgeum-eup, Yangsan, Gyeongsangnam-do 626770, \\ Republic of Korea \\ ${ }^{3}$ College of Korean Medicine, Kyung Hee University, 26 Kyungheedae-ro, Dongdaemun-gu, Seoul 02447, Republic of Korea \\ ${ }^{4}$ Department of Convergence Korean Medical Science, College of Korean Medicine, Kyung Hee University, 26 Kyungheedae-ro, \\ Dongdaemun-gu, Seoul 02447, Republic of Korea \\ ${ }^{5}$ Herbal Medicine Resources Research Center, Korea Institute of Oriental Medicine (KIOM), 111 Geonjae-ro, Naju-si, \\ Jeollanam-do 58245, Republic of Korea \\ ${ }^{6}$ University of Science and Technology (UST), Korean Convergence Medicine Major KIOM, 1672 Yuseongdae-ro, Yuseong-gu, \\ Daejeon 34054, Republic of Korea \\ ${ }^{7}$ Natural Chemotherapeutics Research Institute (NCRI), Ministry of Health, P.O. Box 4864, Kampala, Uganda \\ ${ }^{8}$ Herbal Medicine Research Division, Korea Institute of Oriental Medicine (KIOM), 1672 Yuseong-daero, Yuseong-gu, \\ Daejeon 34054, Republic of Korea
}

Correspondence should be addressed to Woong Mo Yang; wmyang@khu.ac.kr and Youngmin Kang; ymkang@kiom.re.kr

Received 17 February 2020; Accepted 1 April 2020; Published 25 April 2020

Guest Editor: Reggiani Vilela Gonçalves

Copyright (C) 2020 Dae Uk Kim et al. This is an open access article distributed under the Creative Commons Attribution License, which permits unrestricted use, distribution, and reproduction in any medium, provided the original work is properly cited.

\begin{abstract}
This present study evaluated the effects of processed P. multiflorum on osteogenesis using Sarcoma osteogenic (SaOS-2) cell lines and osteoclastogenesis of bone marrow-derived macrophage cells (BMM) and to elucidate differences in effect on the expression of bone-related proteins between commercially sold P. multiflorum and patented, in vitro-propagated Korea Institute of Oriental Medicine (KIOM) P. multiflorum. Raw P. multiflorum and P. multiflorum that were stir-baked and steamed in black bean juice were compared, and western blotting analysis was performed to investigate the expression of bone remodeling-related proteins in SaOS-2 cells. In the cells treated with P. multiflorum steamed in black bean juice, the expression of RANKL was decreased, whereas that of osteoprotegerin, alkaline phosphatase, Runx2, and osterix was increased. Owing to these results, we conclude that processed $P$. multiflorum can be used as an alternative treatment for bone diseases such as osteoporosis, osteopenia, periodontitis, and Paget's disease.
\end{abstract}

\section{Introduction}

In bone metabolism, bones are constantly remodeled by balancing osteoblasts and osteoclasts [1]. In fact, an imbalance between osteoblasts and osteoclasts causes bone loss that can result in various bone diseases such as osteoporosis, osteopenia, periodontitis, and Paget's disease $[2,3]$. In recent years, herbal medicines have been used to increase osteoblast differentiation and decrease osteoclast differentiation for treating bone diseases, including osteoporosis [4-6].

The root tuber of Polygonum multiflorum Thunb. which belongs to the Polygonaceae family, is a medicinal herb that has been used in Traditional Korean Medicine (TKM) as a blood-tonifying medicine [7]. In Dong-Eue-Bo-Gam, which is the TKM book, P. multiflorum is regarded as a medicinal plant with many therapeutic effects, including bone-strengthening 
[8], potent antiaging, and cognitive-enhancing effects [9], as well as the ability to protect human foreskin melanocytes from oxidative stress and improve pigmentation in hair follicles [10]. Polygonum multiflorum also exerts beneficial effects on hippocampal neurons [11].

In TKM, $P$. multiflorum is used after processing because processed $P$. multiflorum exerts better effects than the raw plant $[12,13]$. The processing of herbal medicines, one of the core theories in TKM, is aimed at reducing their toxicity and increasing their beneficial effects [14]. To optimize the utilization of $P$. multiflorum in TKM, the Korea Institute of Oriental Medicine (KIOM) developed and patented a standard protocol for rapid in vitro production of its seedlings and enlarged root tubers [15]. Therefore, the current study aimed to evaluate the potential effects of $P$. multiflorum produced using the patented protocol Korean Patent submission (no: 10-2019-0120751, September 30, 2019) of KIOM and commercially sold P. multiflorum on osteogenesis using osteoblast-like cells Saos-2 and osteoclastogenesis using BMM; this study will serve as a foundation for the use of $P$. multiflorum as a possible treatment of osteoporosis.

\section{Materials and Methods}

2.1. Preparation of Commercially Obtained P. multiflorum. The $2 \mathrm{~kg}$ of the roots of $P$. multiflorum were purchased from Jirisan Hasuo Farming Co. (Sancheong, South Korea) and authenticated by Dr. Kang at Korea Institute of Oriental Medicine (Figure 1(a)) as Commercial Raw P. multiflorum (C-RPM), and $1 \mathrm{~kg}$ dried roots of raw P. multiflorum was baked in a pan with constant stirring at $160^{\circ} \mathrm{C}$ for $40 \mathrm{~min}$ and then maintained in dryer at $45^{\circ} \mathrm{C}$ for 4 hours (Figure 1(b)) as Commercial Stir-Baked to Yellow $P$. multiflorum (C-SBYPM). The $1 \mathrm{~kg}$ of black bean was purchased from Kwangmyongdang Co. (Ulsan, South Korea) and prepared using the method previously suggested [16] in which the obtained beans were boiled in $5 \mathrm{~L}$ of water at $100^{\circ} \mathrm{C}$ for 4 hours to obtain the black beans liquid extract. Then, 4 litres of water was added to the cooked beans and again boiled at $100^{\circ} \mathrm{C}$ for 3 hours to obtain the second extract. The first and second extracts were then mixed to make the black bean juice. $250 \mathrm{~g}$ of $P$. multiflorum and the black bean extract were mixed together in a pot and stirred constantly for 2 hours, then steamed at $60^{\circ} \mathrm{C}$ for 1 hour, and maintained in an oven at $45^{\circ} \mathrm{C}$ for 8 hours (Figure 1(c)) as commercial Steamed with Black Bean Juice P. multiflorum (C-SBBJPM).

2.2. Preparation of KIOM-Obtained P. multiflorum. Raw $P$. multiflorum root tubers produced by the Korean patent protocol (patent no. 10-1777833) were provided by Dr. Kang of Korea Institute of Oriental Medicine and were prepared by the same method as in Section 2.1 as KIOM Raw $P$. multiflorum (K-RPM) (Figure 1(d)), KIOM Stir-Baked to Yellow P. multiflorum (K-SBYPM) (Figure 1(e)), and as KIOM Steamed with Black Bean Juice P. multiflorum (KSBBJPM) (Figure 1(f)).
2.3. Extraction of P. multiflorum. The $10 \mathrm{~g}$ of each of the six $P$. multiflorum mentioned above was extracted using $100 \mathrm{~mL}$ of distilled water for $2 \mathrm{~h}$ at $20^{\circ} \mathrm{C}$ at $200 \mathrm{rpm}$. After filtration, the obtained extracts were concentrated in a vacuum evaporator and powdered by using a freeze-drying machine for $72 \mathrm{~h}$ at $-80^{\circ} \mathrm{C}$. The dried powder weights of C-RPM, C-SBYPM, C-SBBJPM, K-RPM, K-SBYPM, and K-SBBJPM were $2.56 \mathrm{~g}, 2.33 \mathrm{~g}, 1.55 \mathrm{~g}, 1.37 \mathrm{~g}, 1.95 \mathrm{~g}$, and 2.37 g (yield: $25.6 \%, 23.3 \%, 15.5 \%, 13.7 \%, 19.5 \%$, and $23.7 \%$, respectively). The sample was stored at $-20^{\circ} \mathrm{C}$ prior to further studies.

2.4. SaOS-2 Cells Culture. Human osteoblast-like SaOS-2 cells, which have similar properties to primary osteoblasts, were obtained from Seoul National University cell bank (Seoul, South Korea). They were derived from an osteosarcoma and cultured in Dulbecco's Modified Eagle Medium (DMEM) supplemented with $10 \%$ fetal bovine serum and $1 \%$ penicillin at $37^{\circ} \mathrm{C}, 95 \%$ humidity, and $5 \%$ $\mathrm{CO}_{2}$. SaOS- 2 cells were seeded at a density of $1 \times 10^{6}$ cells/ well in 24 -well culture plates. After $24 \mathrm{~h}$ of cultivation, the medium was replaced with $3 \mathrm{~mL}$ medium supplemented with samples. The sample was diluted with the cell culture medium to obtain concentrations of $100 \mu \mathrm{g} / \mathrm{mL}$.

BMM were cultured in an $\alpha$-MEM medium having $10 \%$ fetal bovine serum and $1 \%$ penicillin/streptomycin. BMM were seeded at a density of $1 \times 10^{4}$ cells/well in 96-well culture plates with M-CSF $(60 \mathrm{ng} / \mathrm{mL})$. After 2 hour preincubation with each sample $(50 \mu \mathrm{g} / \mathrm{mL})$ on BMM, RANKL $(100 \mathrm{ng} / \mathrm{mL})$ were treated for 6 days.

2.5. Western Blot Assay. Western blot analysis was performed to investigate the effects of bone metabolism in SaOS-2 cells, osteoclast differentiation factor; RANKL and OPG, osteoblastogenesis factors; alkaline phosphatase (ALP), Runt-related transcription factor 2 (Runx2), and Osterix expressions. Each sample of SaOS-2 cells was vortexed in RIPA buffer (Thermo scientific, Rockford, USA). $20 \mu \mathrm{g}$ cell protein from SaOS- 2 was denatured with $5 \%$ SDS buffer. The prepared protein samples were loaded on $10 \%$ polyacrylamide gels, separated by electrophoresis, and then electrotransferred to activated polyvinylidene fluoride (PVDF) membranes. Membranes were blocked by $3 \%$ bovine serum albumin (BSA) in tris-buffered saline (TBS) containing $1 \%$ Tween 20 (TBS-T) and incubated with the specific antibodies at $4^{\circ} \mathrm{C}$ for $12 \mathrm{~h}(\beta$-actin, OPG, RANKL, Osterix, ALP, RUNX2; Santa Cruz Biotechnology, Inc., CA), $1: 1000$ dilutions in TBS-T). After washing of the membranes for 10 min 3 times, membranes were incubated with anti-rabbit and anti-mouse alkaline phosphataseconjugated secondary antibody $(1: 2000$ dilution in TBS-T) for $1 \mathrm{~h}$ at $24^{\circ} \mathrm{C}$. The membranes were washed three times in TBS-T for 3 min and then reacted with HRP-polymerized secondary antibody for 60 minutes, after completion of the reaction, using a chemiluminescent detection system (Phototope ${ }^{\circledR}$-HRP western blot assay Detection kit, New England Biolab). 


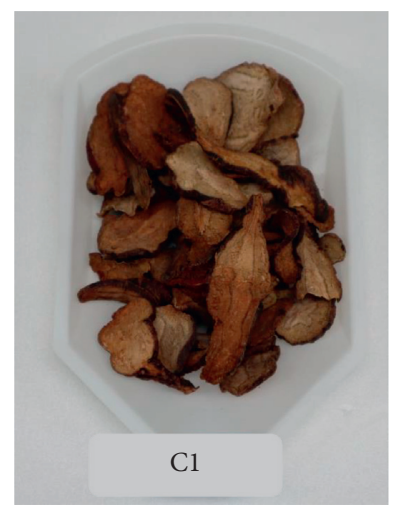

(a)

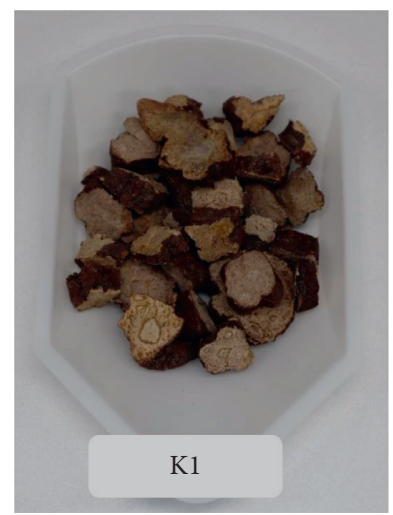

(d)

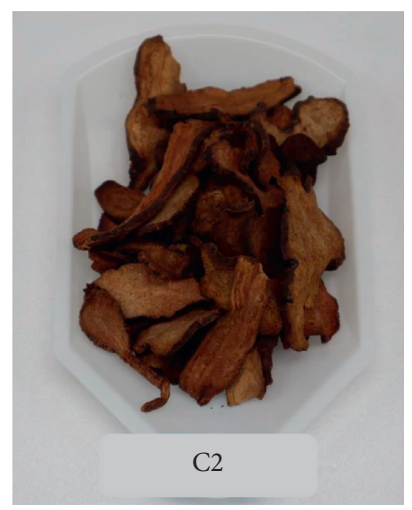

(b)

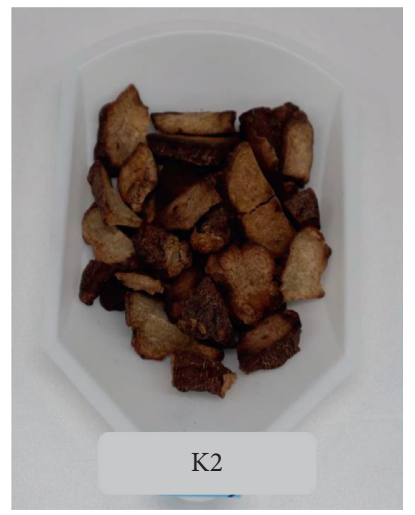

(e)

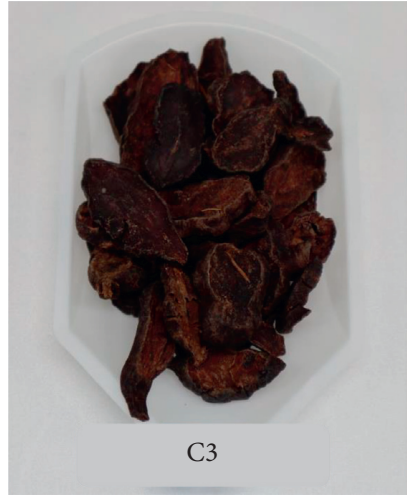

(c)

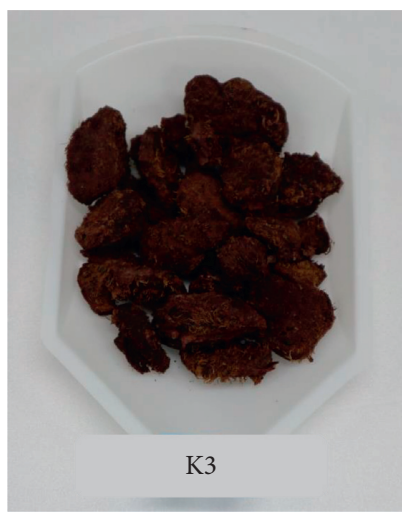

(f)

FIgURE 1: Raw, stir-baked to yellow, and steamed with black bean juice P. multiflorum. (C1 C3: commercial, K1 K3: KIOM product). (a) commercial raw P. multiflorum, C-RPM (C1), (b) commercial stir-baked to yellow P. multiflorum, C-SBYRM (C2), (c) commercial steamed with black bean juice P. multiflorum, C-SBBJPM (C3), (d) KIOM Raw P. multiflorum, K-RPM (K1), (e) KIOM stir-baked to yellow P. multiflorum, K-SBYPM (K2), and (f) KIOM steamed with black bean juice P. multiflorum, K-SBBJPM (K3).

2.6. MTT Assay. The cell viability was assessed using the 3 (4,5-dimethylthiazol-2-yl)-2,5-diphenyl tetrazolium bromide (MTT) solution. For a short time, $1 \times 10^{5}$ cells/well were seeded in 96-well plates and allowed to adhere for $12 \mathrm{~h}$. The cells were treated with $100 \mu \mathrm{L}$ of $100 \mu \mathrm{g} / \mathrm{mL}$ samples each for 24 hours, and MTT $(2 \mathrm{mg} / \mathrm{mL})$ was added $50 \mu \mathrm{L} /$ well and incubated for $2 \mathrm{~h}$, followed by solubilization of the formazan crystals by adding dimethyl sulfoxide (SigmaAldrich, Seoul, South Korea) $50 \mu \mathrm{L} /$ well for $30 \mathrm{~min}$. The color developed was read as optical density using a Gene5 (Biotek, Seoul, South Korea) at $570 \mathrm{~nm}$.

2.7. Statistical Analysis. All data were expressed as a means \pm standard error of the mean. One-way ANOVA followed by the Tukey test (compare all pairs of columns) was used for the statistical analysis. In all analyses, $p<0.05$ was taken to indicate statistical significance.

\section{Results}

3.1. Effects of samples on osteoclast differentiation mediators, RANKL, and OPG in SaOS-2 cells. C-RPM, K-RPM, C-SBYPM, and K-SBYPM were not effective on the decrease of the RANKL expression. On the other hand, C-SBBJPM and K-SBBJSPM treated with black bean juice decreased the expression level of RANKL by $62.3 \%$ and $60.2 \%$, respectively, compared to the control group. C-SBBJPM significantly decreased RANKL expression compared to C-RPM and C-SBYPM. K-SBBJPM significantly decreased RANKL expression compared to K-RPM and K-SBYPM (Figure 2(a)).

C-RPM and K-RPM were not effective on the increase of OPG expression in SaOS-2 cells. On the other hand, C-SBYPM and K-SBYPM and C-SBBJPM and K-SBBJPM increased the level of OPG by $62.7 \%, 90.6 \%, 131.2 \%$, and $153.1 \%$, respectively, compared to the control group. C-SBYPM and C-SBBJPM increased OPG expression compared to C-RPM. C-SBBJPM increased the OPG expression compared to C-SBYPM. Likewise, K-SBYPM and $\mathrm{K}-\mathrm{SBBJPM}$ increased the OPG expression compared to K-RPM. K-SBBJPM increased the OPG expression in SaOS2 cells compared to K-SBYPM (Figure 2(b)).

3.2. Effects of Samples on Osteoblast Differentiation Mediators, ALP, Runx2, and Osterix in SaOS-2 Cells. C-RPM and $\mathrm{K}-\mathrm{RPM}$ and C-SBYPM and K-SBYPM were not effective on the increase of ALP expression in SaOS-2 cells. On the other hand, C-SBBJPM and K-SBBJPM increased the level of ALP 

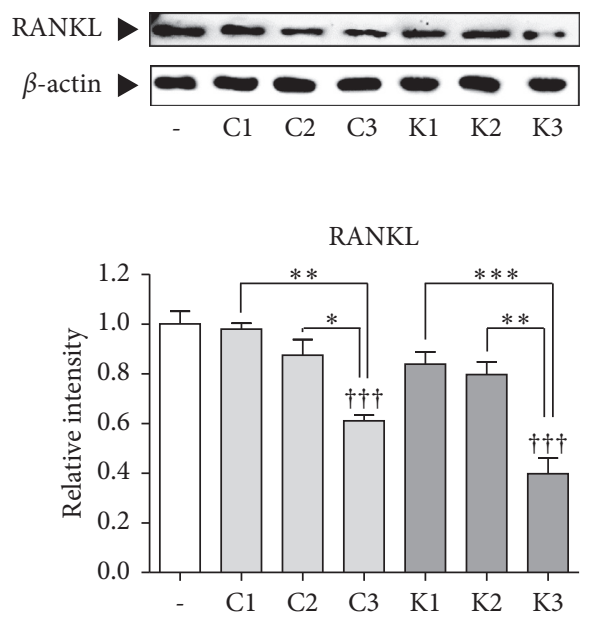

(a)

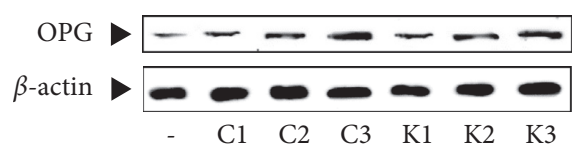

OPG

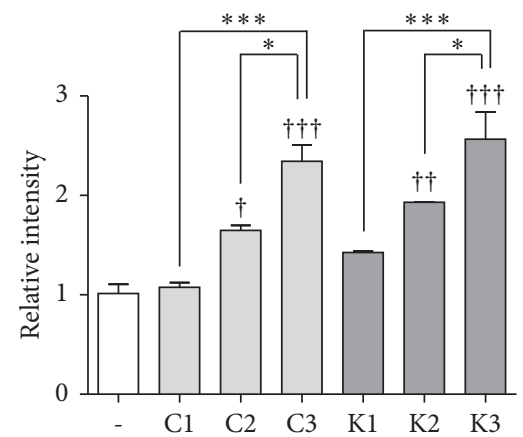

(b)

FIGURE 2: Effects of samples on osteoclast differentiation mediators, RANKL, and OPG in SaOS-2 cells. (a). Effects of samples on RANKL protein levels in SaOS-2 osteosarcoma and expression of RANKL, osteoclast differentiation factor, in SaOS-2 osteosarcoma cells. (b). Effects of samples on OPG protein levels in SaOS-2 osteosarcoma. Expression of OPG, RANKL inhibitor, in SaOS-2 osteosarcoma cells ${ }^{\dagger} p<0.05$, ${ }^{\dagger \dagger} p<0.01$ and ${ }^{\dagger \dagger \dagger} p<0.001$ vs. nontreated cells. ${ }^{*} p<0.05$ and ${ }^{* * *} p<0.001$.

by $81.9 \%$ and $74.6 \%$, respectively, compared to the control group. C-SBBJPM significantly increased the ALP expression compared to C-RPM and C-SBYPM. K-SBBJPM significantly increased the ALP expression compared to K-RPM and K-SBYPM (Figure 3(a)).

C-RPM and K-RPM did not change the expression of Runx2 in SaOS-2 cells. On the other hand, C-SBYPM and K-SBYPM and C-SBBJPM and K-SBBJPM increased the Runx2 expressions by $32.8 \%, 38.3 \%, 41.3 \%$, and $49.0 \%$, respectively, compared to the control group. C-SBYPM and C-SBBJPM increased the Runx2 expression compared to C-RPM. K-SBYPM and K-SBBJPM increased the Runx2 expression compared to K-RPM (Figure 3(b)).

C-RPM and K-RPM and C-SBYPM and K-SBYPM did not increase the expression of Osterix. On the other hand, C-SBBJPM and K-SBBJPM increased the expression of Osterix by $65.5 \%$ and $79.3 \%$, respectively, compared to the control group. C-SBBJPM significantly increased the Osterix expression compared to C-RPM and C-SBYPM. K-SBBJPM significantly increased the Osterix expression compared to K-RPM and K-SBYPM (Figure 3(c)).

3.3. Difference between Commercial P. multiflorum Samples and KIOM Samples. There was no difference of RANKL and OPG expression between commercial P. multiflorum samples and KIOM samples. In addition, there was no difference of ALP, Runx2, and osterix expression between commercial P. multiflorum samples and KIOM samples.

3.4. Effect of P. multiflorum on SaOS-2 Cells Toxicity. The effect of $P$. multiflorum on toxicity in SaOS-2 cells was examined by the MTT assay. The cells were treated with different doses of $P$. multiflorum, and no toxicity was found (Figure 4).

\section{Discussion}

The balance between osteoblasts and osteoclasts is one of the key components of bone metabolism [17]. Bone homeostasis cannot be maintained if there is excessive osteoclastic bone resorption or insufficient osteoblastic bone formation [18]. Attenuation of RANKL or activation of OPG (RANKL inhibitor) might be helpful to inhibit the differentiation of osteoclasts, resulting in alleviation of bone resorption [2]. In this study, the expression of RANKL was significantly decreased by C-SBBJPM and K-SBBJPM treatment, while C-RPM, C-SBYPM and K-RPM, K-SBYPM did not change the expression of RANKL in SaOS-2 cells. This result showed that steamed black bean juice of $P$. multiflorum apparently affected the production of RANKL in osteoblasts. In terms of OPG, all processed $P$. multiflorum samples including C-SBYPM, C-SBBJPM, K-SBYPM, and K-SBBJPM except C-RPM and K-RPM were effective on the expression of OPG in SaOS-2 cells. Nevertheless, steamed black bean juice of $P$. multiflorum was more effective than that of stir-baked to yellow $P$. multiflorum in both of commercial $P$. multiflorum samples and KIOM samples. Steamed black bean juice of $P$. multiflorum increases the inhibitory effects of $P$. multiflorum on bone resorption by osteoblasts by decreasing RANKL expression and increasing OPG expression.

At the stage of osteoblast differentiation, ALP, Runx2 and Osterix, and osteoblast-specific transcription factors are released from osteoblasts, leading to induction of bone formation [2]. This experiment showed that the levels of ALP and Osterix were significantly increased in C-SBBJPM and K-SBBJPM-treated cells. In part of Runx2 expression, C-SBYPM, C-SBBJPM, K-SBYPM, and K-SBBJPM increased the Runx2 compared to raw C-RPM and K-RPM. However, the level of Runx2 in cells treated with C-SBBJPM and K-SBBJPM was higher than C-SBYPM and K-SBYPM. The steamed with black bean juice method apparently 

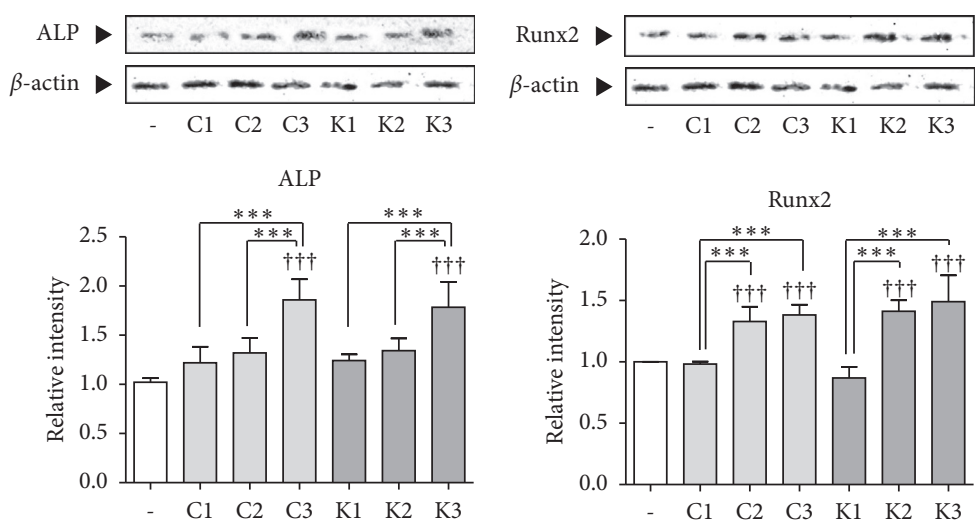

(a)

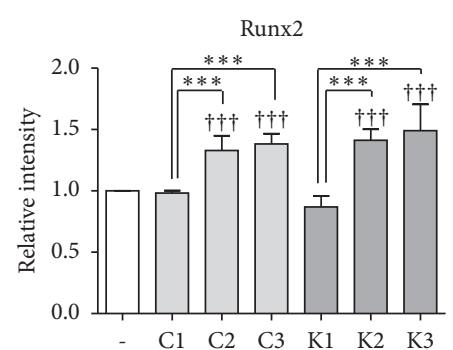

(b)
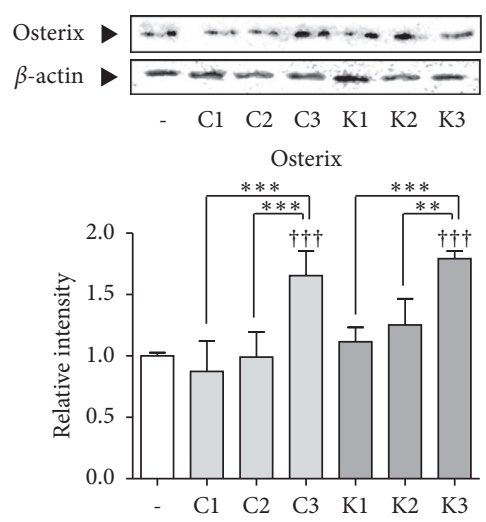

(c)

FIgURE 3: Effects of samples on osteoblast differentiation mediators, ALP, Runx2, and Osterix in SaOS-2 cells. (a) Effects of samples on ALP protein levels in SaOS-2 osteosarcoma. Expression of ALP, osteoblast differentiation factor, in SaOS-2 osteosarcoma cells. (b) Effects of samples on Runx2 protein levels in SaOS-2 osteosarcoma. Expression of Runx2, osteoblast differentiation factor, in SaOS-2 osteosarcoma cells. (c). Effects of samples on Osterix protein levels in SaOS-2 osteosarcoma. Expression of Osterix, osteoblast differentiation factor, in SaOS-2 osteosarcoma cells. ${ }^{\dagger} p<0.05$ and ${ }^{\dagger \dagger} p<0.001$ vs. nontreated cells. ${ }^{* *} p<0.01$ and ${ }^{* * *} p<0.001$.

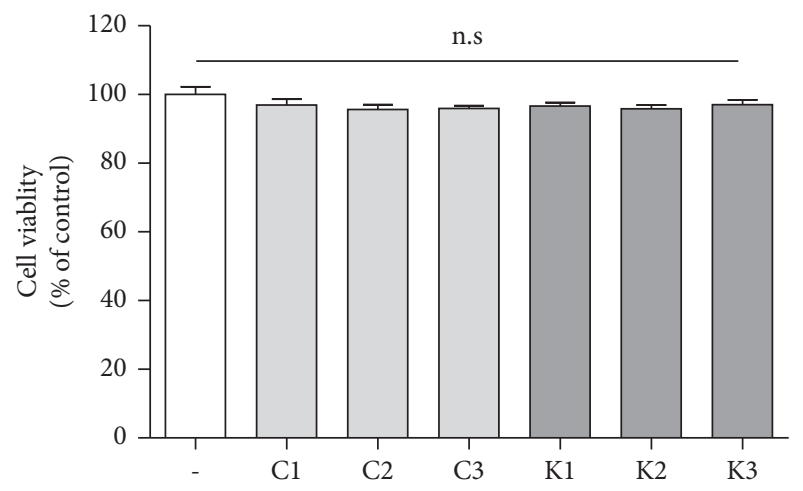

FIGURE 4: Cell viability of SaOS-2 osteosarcoma cells. Cell viability of SaOS-2 osteosarcoma cells. n.s-no significance.

upregulated the effects of $P$. multiflorum on osteoblast activation rather than the stir-baking method which is consistent with the result from osteoclast-related factors.

The cultivation of $P$. multiflorum typically takes long time to propagate which leads to decreasing the yield and quality [19]. However, with the KIOM-patented root tuber enlargement protocol for $P$. multiflorum, rapid in vitro propagation can be carried out without compromising its chemical composition [15]. This experiment shows that the overall tendency of expressions of bone remodeling-related factors between commercial $P$. multiflorum samples and KIOM patented samples is very similar. To clarify the pharmacological potential of K-RPM and K-SBBJPM against bone disease, we are currently investigating the in vivo efficacy of the samples in preclinical animal models.

\section{Conclusion}

Processed P. multiflorum regulated osteoclast differentiation mediators, RANKL, and OPG and increased osteoblast differentiation mediators, ALP, Runx2, and osterix. In addition, there was no significant difference in expressions of RANKL, OPG, ALP, Runx2, and osterix between commercial P. multiflorum samples and KIOM samples. However, although this study has showed promising evidence that processed $P$. multiflorum might be considered as a possible treatment for bone diseases, there is need for further preclinical and clinical studies to enhance future drug development from it for the treatment of bone diseases. Additionally, future study should also consider comparing the phytochemical compositions in the different $P$. multiflorum roots.

\section{Data Availability}

The data for this current study are available from the corresponding author upon reasonable request.

\section{Conflicts of Interest}

The authors declare that there are no conflicts of interest.

\section{Authors' Contributions}

DK, YGK, and RK were involved in material preparation and manuscript writing. JYC, SCJ, MHK, KSS, and WMY conducted the experiments. YMK conceived the original research plans and design of the study. All authors read and approved the final version of the manuscript.

\section{Acknowledgments}

This work was supported by URP (Undergraduate Research Program) of KIOM (C18050) in 2018 and Development of Foundational Techniques for the Domestic Production of Herbal Medicines (K18405), Application of Development of Standardized Herbal Resources (KSN1911420), Korea Institute of Oriental Medicine, through the Ministry of Science and ICT, Republic of Korea. 


\section{References}

[1] N. K. Im, H. J. Kim, M. J. Kim, E. J. Lee, H. I. Kim, and I. S. Lee, "Effects of medicinal herb extracts on osteoblast differentiation and osteoclast formation," Korean Journal of Food Science and Technology, vol. 42, no. 5, pp. 637-642, 2010.

[2] J. E. Lee, M. H. Kim, Y. Y. Choi, H. J. Lee, and W. M. Yang, "Regulation of osteoclastic and osteoblastic differentiation marker expressions in osteoblast-like SaOS-2 cells by Eucommia ulmoides," Oriental Pharmacy and Experimental Medicine, vol. 16, no. 1, pp. 53-57, 2016.

[3] M. B. Greenblatt, J. N. Tsai, and M. N. Wein, "Bone turnover markers in the diagnosis and monitoring of metabolic bone disease," Clinical Chemistry, vol. 63, no. 2, pp. 464-474, 2017.

[4] K. I. Park and J. Oh, "Effects of Liriopis tuber water extract on RANKL-induced osteoclast differentiation," Korean Journal of Physical Anthropology, vol. 24, no. 4, pp. 237-244, 2011.

[5] S. C. Kwak, S. Y. Moon, H. B Kwack et al., "Effect of drynariae rhizoma in RANKL-induced osteoclast differentiation," Korean Journal of Oriental Physiology and Pathology, vol. 26, pp. 506-510, 2012.

[6] Y. H. Choi, J. H. Song, S. J. Jang, J. K. Kim, and M. K. Choi, "Effects of Gastrodia elata blune water extract on RANKLinduced osteoclast differentiation," Korean Journal of Oriental Physiology and Pathology, vol. 24, pp. 807-813, 2010.

[7] G. Narasimhulu, K. K. Reddy, and J. Mohamed, "The genus Polygonum (polygonaceae): an ethnopharmacological and phytochemical perspectives-review," International Journal of Pharmacy and Pharmaceutical Sciences, vol. 6, no. 2, pp. 2145, 2014.

[8] C. Han, "A retrospective analysis of liver function test in patients taking Heshouwu," Korean Oriental Internal Medicine, vol. 31, no. 1, pp. 113-117, 2010.

[9] P. Zhang, X. Tang, and J. Piao, "Effect of polygonum multiflorum thunb on antiaging and enhancing cognition function in D-gal-induced-rats," Chinese Journal of Rehabilitation Medicine, vol. 20, no. 4, pp. 251-253, 2005.

[10] P. Sextius, R. Betts, I. Benkhalifa et al., "Polygonum multiflorum Radix extract protects human foreskin melanocytes from oxidative stress in vitro and potentiates hair follicle pigmentation ex vivo," International Journal of Cosmetic Science, vol. 39, no. 4, pp. 419-425, 2017.

[11] S. M. Ahn, Y. R. Kim, H. N. Kim, H. K. Shin, and B. T. Choi, "Beneficial effects of Polygonum multiflorum on hippocampal neuronal cells and mouse focal cerebral ischemia," The American Journal of Chinese Medicine, vol. 43, no. 4, pp. 637-651, 2015.

[12] X. Wu, X. Chen, Q. Huang, D. Fang, G. Li, and G. Zhang, "Toxicity of raw and processed roots of Polygonum multiflorum," Fitoterapia, vol. 83, no. 3, pp. 469-475, 2012.

[13] C.-e. Zhang, M. Niu, Q. Li et al., "Urine metabolomics study on the liver injury in rats induced by raw and processed Polygonum multiflorum integrated with pattern recognition and pathways analysis," Journal of Ethnopharmacology, vol. 194, pp. 299-306, 2016.

[14] Z. Liang, H. Chen, Z. Yu, and Z. Zhao, "Comparison of raw and processed Radix Polygoni Multiflori (heshouwu) by high performance liquid chromatography and mass spectrometry," Chinese Medicine, vol. 5, no. 1, pp. 1-9, 2010.

[15] Y. Kang, K. Lee, J. Choi et al., "Maximizing seedling and root tuber production in Polygonum multiflorum for use in ethnomedicine," South African Journal of Botany, vol. 119, pp. 119-131, 2018.
[16] W. Zhai, S. Wang, Y. Xiao, and S. Liang, "Study on HPLC specific chromatograms of the processed polygonum multiflorum pieces," Guangdong Chemical Industry, vol. 44, no. 5, pp. 10-12, 2017.

[17] T. C. Phan, J. Xu, and M. H. Zheng, "Interaction between osteoblast and osteoclast: impact in bone disease," Histology and Histopathology, vol. 19, no. 4, pp. 1325-1344, 2004.

[18] K. H. Yoon, J. M. Baek, J. Y Kim et al., "Inhibitory effect on RANKL-induced osteoclast differentiation by water extract of Zizyphus jujuba mill," Korean Journal of Oriental Physiology \& Pathology, vol. 28, pp. 29-34, 2014.

[19] H.-P. Huang, J. Wang, L.-Q. Huang, S.-L. Gao, P. Huang, and D.-L. Wang, "Germplasm preservation in vitro of Polygonum multiflorum thunb," Pharmacognosy Magazine, vol. 10, no. 38, pp. 179-184, 2014. 